\title{
Temporal behavior of the inverse spin Hall voltage in a magnetic insulator-nonmagnetic metal structure
}

\author{
M. B. Jungfleisch $*$ A. V. Chumak, V. I. Vasyuchka, A. A. Serga, \\ B. Obry, H. Schultheiss $A$ P. A. Beck, and B. Hillebrands \\ Fachbereich Physik and Forschungszentrum OPTIMAS, \\ Technische Universität Kaiserslautern, 67663 Kaiserslautern, Germany \\ A. D. Karenowska \\ Department of Physics, Clarendon Laboratory, University of Oxford, OX1 3PU Oxford, United Kingdom \\ E. Saitoh \\ Institute for Materials Research, Tohoku University, Sendai 980-8577, Japan
}

(Dated: October 31, 2018)

\begin{abstract}
It is demonstrated that upon pulsed microwave excitation, the temporal behavior of a spin-wave induced inverse spin Hall voltage in a magnetic insulator-nonmagnetic metal structure is distinctly different from the temporal evolution of the directly excited spin-wave mode from which it originates. The difference in temporal behavior is attributed to the excitation of long-lived secondary spin-wave modes localized at the insulator-metal interface.
\end{abstract}

Over the last decade, the field of spintronics has risen to some prominence. Spintronics is concerned with the development of devices which exceed the performance and energy efficiency of conventional charge-based electronics by exploiting the electron's spin degree of freedom [1-4]. The information currency in spintronic systems is spin angular momentum. Traditional spintronic architectures rely on the electron based spin-transport, however, spin angular momentum can also be transferred by magnons, the quanta of spin waves (collective excitations of the spin lattice of a magnetic material). Magnon physics opens doors to insulator-based spintronic devices which operate with pure spin currents entirely decoupled from charge carriers [1, 5]. Spin waves in magnetic insulators can propagate over macroscopic distances many orders of magnitude longer than the spin diffusion lengths typical of metallic and semiconductor materials [6] 8 . Spin pumping (which transforms spin waves into spin polarized electron currents) and the inverse spin Hall effect (iSHE) (which converts spin polarized electron currents into conventional charge currents) are two physical mechanisms of fundamental importance to the emerging field of 'magnon spintronics'. The temporal characteristics of these phenomena will ultimately determine the operational speeds of magnon spintronic devices [9].

In this Letter we report our findings relating to the temporal behavior of an externally excited spin-wave pulse and a resulting inverse spin Hall voltage in a magnetic insulator-nonmagnetic metal bi-layer. We demonstrate that there are pronounced differences in the temporal evolution of the two signals and show that these differences may be attributed to the excitation of secondary short-wavelength spin-waves localized at the insulatormetal interface via two-magnon scattering of the externally excited mode.

A platinum $(\mathrm{Pt})$ coated yttrium iron garnet (YIG) film

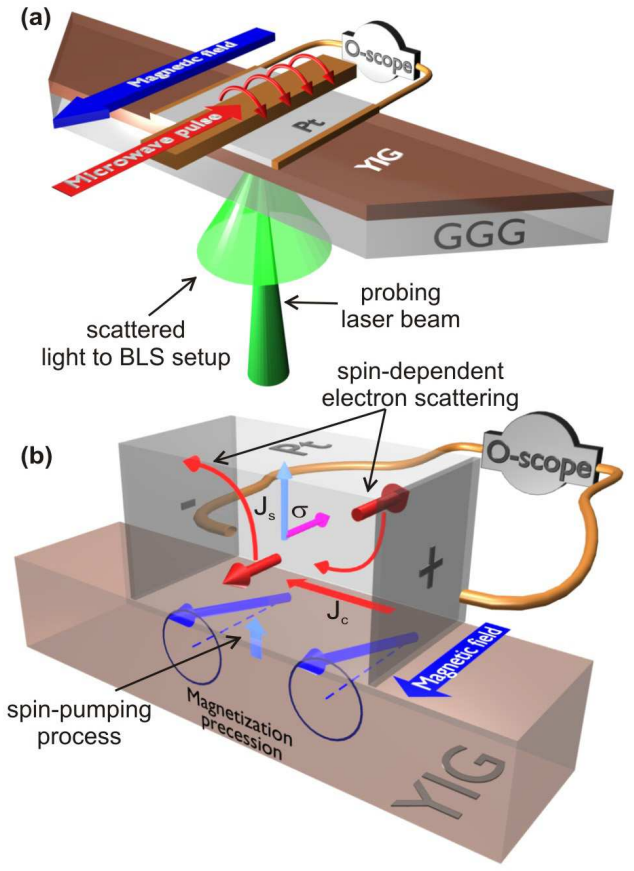

FIG. 1. (Color online) (a) Schematic illustration of the experimental setup. (b) Spin pumping scheme and resulting inverse spin Hall effect.

was used in our experiments. YIG single crystal films have the smallest known spin-wave damping [6, 10]. As a result, magnon currents can be observed in YIG over centimeter distances [1]. Electron scatter in Platinum is strongly spin-dependent making it an attractive material for iSHE voltage generation [12, 13]. The experimental setup is illustrated schematically in Fig. 1(a). It comprises a $2.1 \mu \mathrm{m}$ thick YIG stripe with a $10 \mathrm{~nm}$ thick $3 \times 3 \mathrm{~mm}^{2}$ Pt layer deposited on the top. The edge-to- 


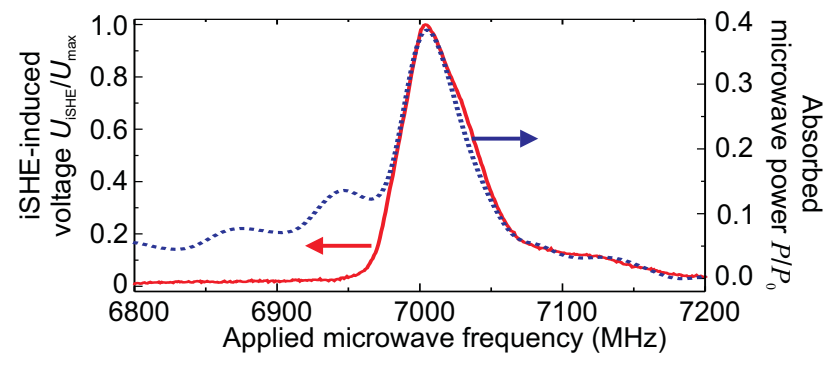

FIG. 2. (Color online) The inverse spin Hall voltage (solid red line) and the absorbed microwave power (blue dashed line) are shown as functions of the applied microwave frequency. Data corresponds to a bias magnetic field of $H_{0}=1820$ Oe. The maximum iSHE voltage $U_{\max }=60 \mu \mathrm{V}$ is observed at the ferromagnetic resonance frequency $f_{0}=7 \mathrm{GHz}$. A decrease in the spin-pumping efficiency for modes excited at frequencies smaller than $f_{0}$ is evident (see Ref. [11]).

edge resistance of the $\mathrm{Pt}$ square is $54 \mathrm{Ohms}$. To ensure good impedance matching and thus minimal distortion of detected signals, the Pt square was connected to the $50 \mathrm{Ohm}$ input of a voltage measuring instrument (see Fig. 1(a)) by a 50 Ohm coaxial cable.

Magnetization precession was excited in the YIG layer by a microwave current applied to a copper microstrip line of width $600 \mu \mathrm{m}$ above the Pt layer. The line was isolated from the Pt by a thin dielectric coating of cyanacrylate. Two excitation geometries were investigated. In the first, the microstrip line was placed across the YIG stripe (Fig. 1(a)), in the second (not shown) it was orientated parallel to it. The temporal behavior of the iSHE voltages observed in the two cases was near identical. Accordingly, we present here only data collected using the first geometry.

The spin-wave modes excited in the YIG film were detected via time-resolved Brillouin light scattering (BLS) spectroscopy [14]. The BLS laser probe beam was focused on the YIG/Pt sample and the intensity of inelastically scattered light (which is directly proportional to the intensity of the scattering spin wave) was analyzed on a $500 \mathrm{ps}$ timescale. For the time-resolved voltage measurements we used a wideband (DC to $200 \mathrm{MHz}$ ) voltage amplifier FEMTO DHPVA-200 and a $300 \mathrm{MHz}$ bandwidth Agilent DSO6034A oscilloscope.

Our experiments were performed in the following fashion: a magnetizing field $\mathbf{H}_{0}$ was applied across the YIG stripe and magnetization precession was driven by a magnetic field $\mathbf{h}(t)$ induced by a microwave current applied to the microstrip line. Under these conditions, due to the spin pumping effect of the precessing magnetization at the YIG/Pt interface, a spin polarized electron current $\mathbf{J}_{\mathrm{S}}$ is produced in the Pt layer [1, 13, 15]. As a consequence of spin-dependent electron scattering in the Pt layer [12] this spin polarized current leads in turn to a conventional charge current $\mathbf{J}_{\mathrm{c}}$ and thus a charge ac- cumulation transverse both to $\mathbf{H}_{0}$ and $\mathbf{J}_{\mathrm{s}}$. Accordingly, an iSHE voltage $U_{\text {iSHE }}$ appears across the Pt square (see Fig. 1(b)). As $\mathbf{J}_{\mathrm{c}} \propto \mathbf{J}_{\mathrm{s}} \times \boldsymbol{\sigma}$ the polarity of the iSHE voltage can be changed by changing the polarization of the spin current $\sigma$ via the static magnetization of the YIG stripe.

In order to increase the dynamic range of the timeresolved iSHE and BLS measurements we supplied a moderately high microwave power to the microstrip line $\left(P_{0}=100 \mathrm{~mW}\right)$. So as to avoid possible caloric effects, driving microwave pulses of $1 \mu \mathrm{s}$ duration were applied with a repetition rate of $10 \mu \mathrm{s}$. The rise and fall times of these pulses were less than 5 ns.

First of all, in order to confirm the origin of the voltage $U_{\text {iSHE }}$ we observed, we verified that its polarity was indeed dependent on the polarity of the magnetization direction. To additionally corroborate our results and to rule out parasitic effects (for example electromagnetic induction in the $\mathrm{Pt}$ stripe) we also tested a structure with a nonmagnetic insulator (gadolinium gallium garnet, GGG) in the place of the YIG. No voltage was detected. Thus, we are able to say with confidence that the voltages we observed were due to the iSHE.

The absorbed microwave power (directly proportional to the intensity of the excited spin waves) and the iSHE voltage were measured as the applied microwave frequency was varied for a magnetizing field $H_{0}=1820 \mathrm{Oe}$ (see Fig. 2). The maximum microwave absorption was recorded for the spin-wave mode excited at the frequency of ferromagnetic resonance (FMR) $f_{0}=7 \mathrm{GHz}$. The observed FMR linewidth was around $50 \mathrm{MHz}$ (corresponding to $2 \Delta H_{\mathrm{FMR}}=17 \mathrm{Oe}$ ). This value is significantly larger than the FMR linewidth of 0.6 Oe quoted by the producers of the YIG film. The apparent discrepancy is due to the fact that in our experiments the FMR mode is strongly coupled to the exciting microstrip line, and thus the dominant dissipative mechanism is not the small magnetic damping but the large radiation loss back into the line.

The $50 \mathrm{MHz}$ bandwidth of the microstrip-loaded FMR was wide enough to contain the frequency spectrum of the driving microwave pulse of $1 \mu$ s duration without significant distortion. In order to determine the distortion level the convolution of the Fourier transformed input pulse and the spectrum of the absorbed microwave power was calculated. Using the inverse Fourier transformation of this convolution, we obtained the calculated 'tailing' of the spin-wave pulse edges, which was smaller than $20 \mathrm{~ns}$. As the measured iSHE voltage originates directly from the spin-wave amplitude at the YIG/Pt interface (Fig. 2) a similar tail was expected to be observed on the $U_{\text {iSHE }}$ pulse. However, the real temporal behavior both of the spin-wave amplitude and iSHE voltage proved to be much more complex. Note that time-resolved BLS measurements performed at $f_{0}=7 \mathrm{GHz}$ established that excitation delays between different sample points were 
always smaller than $1 \mathrm{~ns}$, confirming the excitation of quasi-uniform FMR rather than spin-wave modes traveling away from the microstrip line. Therefore, no time delays associated with a nonzero spin-wave propagation time through the Pt area appear in the iSHE signal.

The time resolved measurements were performed at $f_{0}=7 \mathrm{GHz}$ where the iSHE voltage was maximum. In Fig. 3 the time profiles of the spin-wave and voltage pulses are compared. The spin-wave intensity (blue dashed line in Fig. 3) increases rapidly when the microwave pulse is applied at $t=0 \mathrm{~ns}$. After some oscillations corresponding to a nonlinear transition process (common for relatively high spin-wave intensities [16]), an equilibrium value is reached. When the microwave pulse is switched off at $t=1000 \mathrm{~ns}$, the BLS signal decreases rapidly. The measured iSHE voltage is shown by a red solid line in Fig. 3 It is clear that the rise and fall times of the iSHE voltage are appreciably longer than those corresponding to the spin-wave intensity.

In order to unpick the peculiarities of the temporal evolution of the iSHE signal and to relate these to the dynamics of the precessing magnetization, we show the waveforms plotted on logarithmic scale in Fig. 4. The data of Fig. 4 has several features which warrant clarification:

Firstly, the falling slopes of both the iSHE voltage and of the spin-wave intensity are nonexponential (note the logarithmic scale).

Secondly, the measured iSHE voltage rises and decreases much more slowly than the spin-wave intensity; for example, during the first $50 \mathrm{~ns}$ after the driving microwave pulse has been switched off, the iSHE voltage decreases only by factor of 3 whereas the spin-wave intensity falls by factor of 50 .

Thirdly, for $t>1600$ ns the iSHE voltage and spinwave intensity decay exponentially and their fall times $\tau_{\text {iSHE }}$ and $\tau_{\text {SW }}$ are very similar; $460 \mathrm{~ns}$ and $420 \mathrm{~ns}$ respectively.

These facts can be understood if one assumes that-

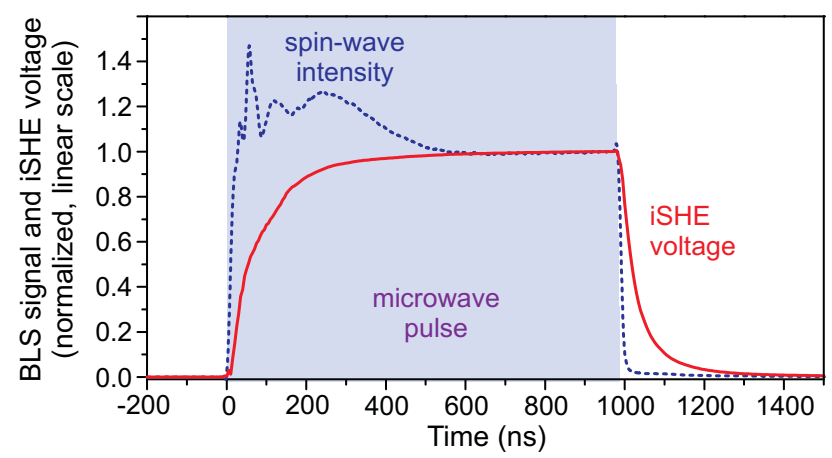

FIG. 3. (Color online) Comparison of the normalized spinwave signal measured with Brillouin light scattering spectroscopy (blue curve) and the iSHE voltage (red curve). The maximum iSHE voltage $U_{\max }$ is $60 \mu \mathrm{V}$.

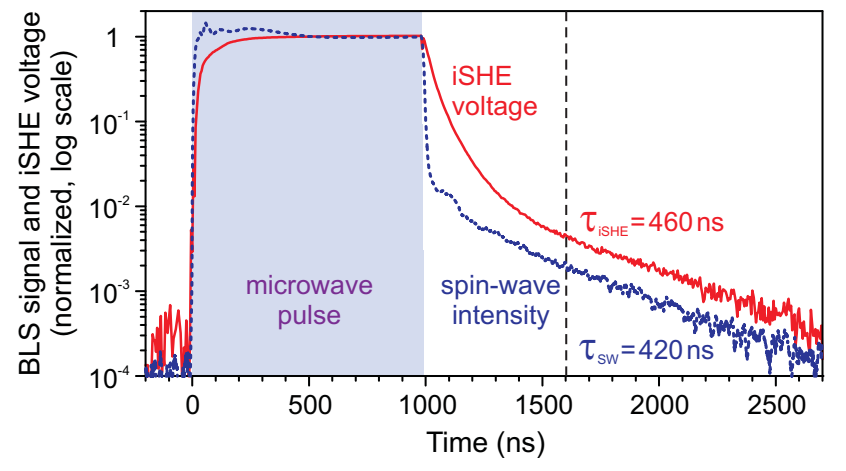

FIG. 4. (Color online) Spin-wave intensity and iSHE voltage as a function of time (logarithmic scale).

rather than solely the externally driven spin-wave mode - many modes contribute to the iSHE voltage. In order to illustrate how such a model fits with the experimental data we consider for simplicity only two groups of modes. The first group corresponds to the FMR directly excited by the microstrip line. This group is characterized by a large amplitude (determined by the applied microwave signal) and a high decay rate due to its strong coupling to the microstrip antenna (see Fig. 2). A second mode group is excited indirectly via two-magnon scattering of the first group by defects and inhomogeneities in the YIG film. This well known mechanism results in the redistribution of energy from the externally excited uniform mode into dipolar-exchange spin-wave (DESW) modes with wavelengths determined by sizes of the scatterers (typically $1 \mu \mathrm{m}$ in high quality YIG samples) [6, 10, 17 19]. Due to their extremely short wavelengths, the DESW modes are entirely decoupled from the microstrip line and their relaxation $\left(2 \Delta H_{\text {DESW }}=0.1-0.2\right.$ Oe $\left.[10,18,19]\right)$ is dominated by the intrinsic magnetic damping of the film [10]. Since the impurities and inhomogeneities are concentrated close to the YIG film surfaces, these modes are localized near the YIG/Pt interface.

The weak DESW group cannot be resolved by BLS on the background of the strong uniform FMR mode (in addition, the BLS setup is less sensitive to short-wavelength spin waves) and thus is visible only after the microwave pulse has been switched off and the fast-relaxing FMR precession has decayed. It follows that the spin-wave fall time $\tau_{\mathrm{SW}}$ of $420 \mathrm{~ns}$ measured for $t>1600 \mathrm{~ns}$ is determined only by the relaxation of the DESW modes. Therefore, for $t>1600 \mathrm{~ns}$, the long-lived DESW modes are the sole contributors to the BLS signal and the iSHE voltage. As a result, the slopes $\tau_{\mathrm{iSHE}}$ and $\tau_{\mathrm{SW}}$ are approximately the same. The value of the fall time in this region of the signal is a direct evidence for the dipolarexchange nature of these waves: it corresponds to a resonance curve linewidth of $2 \Delta H_{\mathrm{DESW}}=0.14$ Oe which fits very well with literature data on DESW relaxation. 
We suggest that the DESW modes, in spite of their small amplitude, make a relatively more significant contribution to the iSHE voltage than the directly excited FMR mode due to their localization close to the YIG/Pt interface. As a result, after the excited spin-wave intensity maximum, the iSHE voltage continues to grow (for times $t<500 \mathrm{~ns})$. The same effect is visible after the microwave pulse has been switched off: the iSHE voltage continues to be generated by the long-lived DESW group.

The model we propose accounts well for the main features of the experimental results even in the case that we consider contributions from only two spin-wave groups to the iSHE voltage signal (the slowest and the fastest relaxing). For quantitative analysis of the transition regions (times $t<500 \mathrm{~ns}$ and $1000 \mathrm{~ns}<t<1600 \mathrm{~ns}$ ) the contributions of spin-wave modes having intermediate life times must be taken into account (for example, in order to fit the entire iSHE slope for time interval $t>1000 \mathrm{~ns}$ three exponential functions are required). Experimentally, these modes can be excited both as a result of elastic two-magnon scattering and due to nonlinear four-magnon scattering of the FMR mode. Additional experiments performed at different microwave powers (in the range from $25 \mathrm{~mW}$ to $1 \mathrm{~W}$ ) showed slight variations of the iSHE slopes for times $t<500 \mathrm{~ns}$ and $1000 \mathrm{~ns}<t<1600$ ns which can be attributed to the effect of nonlinearity. Nevertheless, we emphasize that the qualitative behavior of the voltage pulse as well as its fall time for $t>1600 \mathrm{~ns}$ remains the same regardless of the FMR mode power.

In summary, we report that the iSHE voltage increases and decreases significantly more slowly than the intensity of the pulsed externally driven uniform spin-wave mode from which it originates. From the complex temporal behavior of both signals we have observed we conclude that indirectly excited short-wavelength dipolar-exchange spin waves participate in spin pumping at the metal-insulator interface as well as directly excited uniform precession. The surface localization of the scattered dipolar-exchange modes means that despite their low amplitude and incoherent character, they make a substantial contribution to the iSHE voltage signal. In addition, we can conclude that iSHE voltage signal delays in magnetic insulatornonmagnetic metal structures are dominated by spinwave dynamics in the insulator, rather than electron dynamics in the metal.

We thank G. E. W. Bauer for the valuable discussions and the Nano+BioCenter, TU Kaiserslautern, for technical support. A. D. K. is grateful for the support of Magdalen College, Oxford. B. O. would like to acknowledge the DFG for support within the Graduiertenkolleg 792.

* jungfleisch@physik.uni-kl.de

$\dagger$ Current adress: Materials Science Division and Center for Nanoscale Materials, Argonne National Laboratory, Argonne, Illinois 60439, USA

[1] Y. Kajiwara, K. Harii, S. Takahashi, J. Ohe, K. Uchida, M. Mizuguchi, H. Umezawa, H. Kawai, K. Ando, K. Takanashi, S. Maekawa, and E. Saitoh, Nature 464, 262 (2010).

[2] S. A. Wolf, D. D. Awschalom, R. A. Buhrman, J. M. Daughton, S. von Molnár, M. L. Roukes, A. Y. Chtchelkanova, and D. M. Treger, Science 294, 5546 (2001).

[3] I. Žutic, J. Fabian, and S. Das Sarma, Rev. Mod. Phys. 76, 2 (2004).

[4] J. F. Gregg, Nature Mater. 6, 798 (2007).

[5] K. Uchida, J. Xiao, H. Adachi, J. Ohe, S. Takahashi, J. Ieda, T. Ota, Y. Kajiwara, H. Umezawa, H. Kawai, G. E. W. Bauer, S. Maekawa, and E. Saitoh, Nature Mater. 9, $894(2010)$

[6] A. A. Serga, A. V. Chumak, and B. Hillebrands, J. Phys. D: Appl. Phys. 43, 264002 (2010).

[7] T. Schneider, A. A. Serga, B. Leven, B. Hillebrands, R. L. Stamps, and M. P. Kostylev, Appl. Phys. Lett. 92, 022505 (2008).

[8] A. Khitun, M. Bao, J. Lee, K. Wang, D. W. Lee, and S. Wang, Materials Research 998 (2007).

[9] N.P. Stern, D. W. Steuerman, S. Mack, A.C Gossard, and D.D. Awschalom Nature Physics 4, 843 (2008).

[10] A. G. Gurevich and G. A. Melkov, Magnetization Oscillations and Waves (CRC, New York, 1996).

[11] C. W. Sandweg, Y. Kajiwara, K. Ando, E. Saitoh, and B. Hillebrands Appl. Phys. Lett. 97, 252504 (2010).

[12] J. E. Hirsch, Phys. Rev. Lett. 83, 1834 (1999).

[13] Y. Tserkovnyak, A. Brataas, and G. E. W. Bauer, Phys. Rev. B 66, 224403 (2002).

[14] S. O. Demokritov, B. Hillebrands, and A. N. Slavin, Phys. Rep. 348, 441 (2001).

[15] S. Mizukami, Y. Ando, and T. Miyazaki, Phys. Rev. B 66, 104413 (2002).

[16] V. S. L'vov, Wave Turbulence under Parametric Excitations: Applications to Magnetics (Springer, Berlin, 1994).

[17] M. Sparks, Ferromagnetic Relaxation Theory (McGrawHill, New York, 1964).

[18] G. A. Melkov, V. I. Vasyuchka, Yu. V. Kobljanskyj, and A. N. Slavin, Phys. Rev. B 70, 224407 (2004).

[19] G. A. Melkov, A. D. Dzyapko, A. V. Chumak, and A. N. Slavin, J. Exp. and Theor. Phys. 99, 1193 (2004). 